\title{
Eating Disorders in Sport: Comparing Eating Disorder Symptomatology in Athletes and Non-Athletes During Intensive Eating Disorder Treatment
}

\author{
Laura K. Fewell \\ University of Missouri-St. Louis
}

Riley Nickols and Amanda Schlitzer Tierney

McCallum Place Eating Disorder Treatment Centers

\author{
Cheri A. Levinson \\ University of Louisville
}

\begin{abstract}
The current study tested if athlete patients differed from non-athlete patients in measures of eating disorder (ED) and related pathology. Athlete $(n=91$ in Study 1; $n=39$ in Study 2) and non-athlete ( $n=76$ in Study 1; $n=26$ in Study 2) patients completed self-report measures, and body mass index (BMI) was calculated. Athlete patients had significantly lower ED symptomatology and depression than non-athlete patients $(p s<.05)$. ED impairment, worry, psychosocial functioning, BMI, obsessive-compulsiveness, and compulsive exercise did not significantly differ between groups ( $p s>.08$ ). Greater ED symptomatology was associated with higher psychosocial functioning among athlete patients and higher obsessivecompulsive symptoms and compulsive exercise among non-athlete patients. This is a novel study comparing ED symptomatology and related measures of mental health in athlete and non-athlete patients engaged in residential or partial hospitalization ED treatment. Future research should further investigate how participation in high-level sport impacts the presentation, treatment, and outcome of individuals with EDs.
\end{abstract}

Keywords: athlete, eating disorder, outcomes, sport, treatment

\footnotetext{
Fewell is with the Dept. of Psychological Sciences, University of Missouri-St. Louis, St. Louis, MO. Nickols and Schlitzer Tierney are with The Victory Program, McCallum Place Eating Disorder Treatment Centers, St. Louis, MO. Levinson is with the Dept. of Psychological and Brain Sciences at University of Louisville, Louisville, KW. Address author correspondence to Cheri A. Levinson at cheri.levinson@louisville.edu.
} 
Eating disorders (EDs) are serious illnesses with the highest mortality rate among all psychiatric disorders (Harris \& Barraclough, 1998). The prevalence of EDs has been found to be higher in athletes than non-athletes: non-athletes hold a lifetime prevalence rate of up to $4.6 \%$ (Sundgot-Borgen \& Torstveit, 2004), while the lifetime prevalence rate of EDs among athletes has been shown to be as high as $13.5 \%$ (Sundgot-Borgen, 1993). Yet few studies have investigated whether differences in ED symptoms and related pathology exist between athletes and non-athletes, and no known study has examined comparisons between athlete and non-athlete patients with EDs specifically (Arthur-Cameselle, Sossin, \& Quatromoni, 2017). An improved understanding of any differences in athlete and non-athlete patients with EDs is imperative for tailoring specialized treatment to the needs of each population and for informing ED treatment and recovery in general.

\section{ED Symptoms in Athletes and Non-Athletes}

Studies comparing athletes to non-athletes in the context of ED and related measures are sparse. Several studies have found that athletes have significantly fewer ED symptoms and less body image disturbance than non-athletes (DiBartolo \& Shaffer, 2002; Hulley \& Hill, 2001; Wollenberg, Shriver, \& Gates, 2015), which may be due to the potential protective mental health benefits of sport and exercise (Goodwin, Haycraft, \& Meyer, 2016; Jewett et al., 2014). Yet the comparatively high rates of EDs among athletes suggest that sport may increase rather than decrease ED risk (Gritti et al., 2016).

One explanation for these incongruities may be attributed to how athletes evaluate their ED symptoms. For instance, Thompson and Sherman (2010) found that athletes underreport ED symptoms due to the misperception that food restriction and excessive exercise will enhance sport performance and are not, in fact, problematic. Indeed, certain aspects of sport, such as mental toughness and commitment to training, may exacerbate ED risk and behaviors (i.e., food restriction, excessive exercise), yet these factors are often normalized and reinforced within the sport culture and might not be viewed as problematic (Thompson \& Sherman, 1999; Plateau et al., 2017). Furthermore, several studies have demonstrated that athletes and coaches believe weight loss and thinness can elevate sport performance and are not areas of concern or potential risk (De Bruin, Oudejans, \& Bakker, 2007; Jones, Glintmeyer, \& McKenzie, 2005).

Another explanation for the discrepancies in ED symptoms among athletes and non-athletes may be the underlying basis for ED pathology. For example, fear of weight gain and drive for thinness are defining components of many EDs (Levinson et al., 2017; Penas-Lledo, Bulik, Lichtenstein, Larsson, \& Baker, 2015), and participation in weight-related and aesthetic sports has been more highly associated with ED symptomatology (i.e., symptoms characteristic of EDs) and body dissatisfaction than sport without an emphasis on weight and appearance (Anderson, Reilly, Gorrell, \& Anderson, 2016; De Bruin, Woertman, Bakker, \& Oudejans, 2009; Sundgot-Borgen \& Torstveit, 2004). Because of the paucity in research comparing athletes to non-athletes with ED diagnoses, it is challenging to draw any conclusions as to why differences between these populations exist. 


\section{Mood and Anxiety Disorders}

Other differentiating markers of mental health, such as mood and anxiety disorders, are also poorly understood among athlete and non-athlete populations with EDs. Depression and anxiety are reportedly lower in athletes than non-athletes (Hainline, 2015; Pearlstein, 2016) but higher in individuals with EDs compared to the general population. In an NCAA Sport Science Institute report of 65,026 students, athletes were significantly less likely than non-athletes to report feelings of anxiety and depression; rates were still high, however, with $48 \%$ of female and $31 \%$ of male athletes reporting "overwhelming anxiety" (vs. $56 \%$ of non-athletes), and $28 \%$ of female and $21 \%$ of male athletes reporting depressive symptoms (compared to $33 \%$ of non-athletes; Hainline, 2015). Yet it is unclear if athletes with EDs have lower depression and anxiety than non-athletes with EDs. This uncertainty is exacerbated by the high rate of depression and anxiety among individuals with EDs: as many as $80 \%$ of individuals with EDs have depression (Green et al., 2009; Grilo, White, \& Masheb, 2009) and up to 75\% of women with EDs have anxiety (Aimé, Guitard, \& Grousseaud, 2017), compared to 11\% (Carey et al., 2014) and $18 \%$ in the general population (Kessler et al., 2005) respectively.

Obsessive-compulsive disorder (OCD) has also been studied among ED populations, but prevalence rates have varied, with some studies finding OCD as low as $9.5 \%$ (Godart, Flament, Perdereau, \& Jeammet, 2002) and others as high as $62 \%$ in ED populations (Kaye, Bulik, Thornton, Barbarich, \& Masters, 2004). Rates of OCD in athlete populations are much lower-approximately 5\% (Cromer, Kaier, Davis, Stunk, \& Stewart, 2017)—-though this is higher than the general population prevalence rate of approximately $2 \%$ (Ruscio, Stein, Chiu, \& Kessler, 2010). Notably, rates of OCD among athlete patients and non-athlete patients with EDs have not been studied. Such findings may further inform the specifics of treatment for this population.

\section{Compulsive Exercise}

Compulsive exercise is another common comorbidity in EDs (Davis et al., 1997), yet literature regarding its presentation among athletes versus non-athletes with EDs is sparse and inconsistent. A recent study (Noetel et al., 2016) found that selfidentified athletes (either a current or former athlete) did not differ from nonathletes on the Compulsive Exercise Test (CET; a measure of compulsive exercise specifically in EDs; Taranis, Touyz, \& Meyer, 2011). CET results, however, were significantly associated with ED pathology, depression, anxiety, and OCD. Conversely, Goodwin et al. (2016) found greater levels of compulsive exercise on the CET in adolescent athletes versus non-athletes, while Turton, Goodwin, and Meyer (2017) found a high athletic identity (e.g., degree to which one identifies with the athletic role; Brewer, Van Raalte, \& Linder, 1993; Hughes \& Leavey, 2012) among long-distance runners to be more strongly associated with compulsive exercise, compared to those with a more multifaceted sense of self. Interestingly, athletic identity was not correlated with ED symptomatology. Research has identified compulsive exercise as an important mechanism in the development and maintenance of EDs, and compulsive exercise is associated with increased risk of relapse after treatment (Davis et al., 1997; Davis, Katzman, \& Kirsh, 1999). 
Therefore, replication of previous findings on the CET (Goodwin et al., 2016; Taranis et al., 2011) is warranted. Examining if differences exist in ED and related measures among athlete patient and non-athlete patient populations will allow treatment centers to more effectively adapt treatments to meet the needs of athletes.

\section{Study Aims}

The aim of the current study was to test if athlete patients with EDs differed from non-athlete patients with EDs (i.e., general ED patients who did not participate in high-level, competitive sport) and to replicate CET findings from previous studies (Goodwin et al., 2016; Taranis et al., 2011). In Study 1, ED symptomatology, ED impairment, depression, worry, and BMI (in AN) were measured and differences between athlete patients and non-athlete patients upon treatment admission were evaluated. In Study 2, ED pathology, obsessive-compulsive symptoms, and compulsive exercise were measured and differences were assessed between athlete and non-athlete patients at treatment admission.

\section{Study 1: Methods}

\section{Participants}

Participants were 91 male and female athlete patients and 76 male and female non-athlete patients entering either a residential or partial hospitalization (PHP) treatment program for EDs between December 2012 and June 2017. Participants were diagnosed with anorexia nervosa (AN; restricting or binge/purge subtype), bulimia nervosa $(\mathrm{BN})$, eating disorder not otherwise specified (EDNOS), other specified feeding or eating disorder (OSFED; a diagnosis derived from the DSM-V revision which was used during the latter course of the study), binge-eating disorder (BED; also used in conjunction with the release of the DSM-V), avoidant/restrictive food intake disorder (ARFID; used in conjunction with the release of the DSM-V), or rumination disorder (used in conjunction with the release of the DSM-V). Diagnoses were made by board-certified psychiatrists using semi-structured interviews upon treatment admission. Demographic and clinical information is found in Table 1.

\section{Procedures}

This study was approved by the Institutional Review Board at Washington University in St. Louis. Participants (combined group $N=167$ ) completed the following assessments at the beginning of treatment via paper and pencil: Eating Disorder Examination Questionnaire (EDE-Q; Fairburn, 2008); Clinical Impairment Assessment (CIA; Bohn \& Fairburn, 2008); Beck Depression Inventory II (BDI-II; Beck, Steer, \& Brown, 1996); Penn State Worry Questionnaire (PSWQ; Meyer, Miller, Metzger, \& Borkovec, 1990); and World Health Organization Disability Assessment Schedule 2.0 (WHODAS; Ustün, Kostanjsek, Chatterji, \& Rehm, 2010). Body mass index (BMI) was calculated upon admission by an approved treatment staff member. 


\section{Table 1 Study 1: Demographic and Clinical Characteristics of Athlete and Non-Athlete Patients Using PSM}

\begin{tabular}{|c|c|c|c|}
\hline & Athlete & Non-Athlete & Total \\
\hline & $\mathrm{n}=91$ & $\mathrm{n}=76$ & $n=167$ \\
\hline \multicolumn{4}{|l|}{ Gender, $n(\%)$} \\
\hline Female & $80(87.9)$ & $70(92.1)$ & $150(89.8)$ \\
\hline Male & $11(12.1)$ & $6(7.9)$ & $17(10.2)$ \\
\hline European American, $n(\%)$ & $84(92.3)$ & $70(92.1)$ & $154(92.2)$ \\
\hline \multicolumn{4}{|l|}{ ED Diagnoses, $n(\%)$} \\
\hline $\mathrm{AN}$ & $63(69.2)$ & $50(65.8)$ & $113(67.7)$ \\
\hline $\mathrm{BN}$ & $11(12.1)$ & $12(15.8)$ & $23(13.8)$ \\
\hline EDNOS & $6(6.6)$ & $10(13.2)$ & $16(9.6)$ \\
\hline OSFED & $5(5.5)$ & $2(2.6)$ & $7(4.2)$ \\
\hline BED & $4(4.4)$ & $1(1.3)$ & $5(3.0)$ \\
\hline ARFID & $2(2.2)$ & $0(0)$ & $2(1.2)$ \\
\hline Rumination & $0(0)$ & $1(1.3)$ & $1(.6)$ \\
\hline LOS in days, $M(S D)$ & $66.8(37.8)$ & $52.6(33.9)$ & $60.2(36.7)$ \\
\hline LOS range & 13 to 210 days & 9 to 180 days & 8 to 210 days \\
\hline Age in years, $M(S D)$ & $19.4(3.0)$ & $18.4(4.2)$ & $18.9(3.6)$ \\
\hline Age range & 13 to 30 years & 11 to 32 years & 11 to 32 years \\
\hline Duration of ED in years, $M(S D)$ & $4.3(3.2)$ & $4.4(3.7)$ & $4.3(3.4)$ \\
\hline Duration of ED range & 0 to 15 years & 0 to 15 years & 0 to 15 years \\
\hline Admit $\mathrm{BMI}<18.5, n(\%)$ & $41(45)$ & $36(47)$ & $77(46)$ \\
\hline
\end{tabular}

Note. PSM = Propensity Score Matching; $\mathrm{AN}=$ Anorexia Nervosa; $\mathrm{BN}=$ Bulimia Nervosa; EDNOS = Eating Disorder Not Otherwise Specified; OSFED = Other Specified Feeding or Eating Disorder; $\mathrm{BED}=$ Binge Eating Disorder; ARFID = Avoidant Restrictive Food Intake Disorder; Rumination = Rumination Disorder; LOS = Length of Stay in days for the total number of days in either residential and/or partial hospitalization programming; admit BMI = Admission Body Mass Index.

\section{Outcome Measures}

Eating Disorder Examination Questionnaire 16.0 (EDE-Q; Fairburn, 2008) is a 28-item self-report questionnaire designed to assess ED behaviors and thoughts. The global score of the EDE-Q, calculated by summing subscale totals and dividing by the number of subscales, was used to measure overall eating symptomatology. The EDE-Q has demonstrated excellent test-retest reliability and internal consistency (Luce \& Crowther, 1999), and acceptable to good criterion validity and concurrent validity (Aardoom, Dingemans, Slof Op't Landt, \& Van Furth 2012; Mond, Hay, Rodgers, Owen, \& Beumont, 2004). Cronbach's $\alpha$ was .96 for the present study.

Clinical Impairment Assessment (CIA; Bohn \& Fairburn, 2008) is a 16-item self-report questionnaire to be used in conjunction with the EDE-Q. The CIA assesses ED impairment on mood as well as cognitive and interpersonal functioning. The global score of the CIA was used and was calculated by summing item 
totals. The CIA has demonstrated high internal consistency, test-retest reliability, and good construct and criterion validity (Vannucci et al., 2012). Cronbach's $\alpha$ was .95 for the present study.

Beck Depression Inventory II (BDI-II; Beck et al., 1996) is a 21-item selfreport measure assessing the severity of depression and was used in the current study to measure depression. The BDI-II has demonstrated high internal consistency and concurrent validity (Storch, Roberti, \& Roth, 2004). Cronbach's $\alpha$ was .94 for the present study.

Penn State Worry Questionnaire (PSWQ; Meyer et al., 1990) is a 16-item self-report questionnaire measuring trait assessment of pathological worry. It has demonstrated high internal consistency, high test-retest reliability, and good convergent validity (Stöber, 1998). Cronbach's $\alpha$ was .72 for the present study.

World Health Organization Disability Assessment Schedule 2.0 (WHODAS; Ustün et al., 2010) is a 36-item, self-report measure designed to assess disability across six domains, including areas of communication and participation in society. The global score (scored by summing all items) was used to measure psychosocial functioning. The WHODAS has shown very good internal consistency and good test-retest reliability (Ustün et al., 2010). Cronbach's $\alpha$ was .97 for the present study.

Body Mass Index (BMI) was assessed at treatment admission by an approved staff using a medical grade Detecto precision scale and height tool. Participants were weighed in light clothing and were not informed of their weight.

\section{Analyses}

Propensity score matching (PSM; Rosenbaum \& Rubin, 1983) was used to create a final dataset $(N=167)$ that accounted for the covariates of each treatment group (athlete and non-athlete) for the following variables: length of stay (LOS), ED diagnosis, age, duration of ED, gender, and admission BMI (see Table 1 for variable means). After using PSM, independent sample t-tests were conducted to test for differences in ED symptomatology and impairment, depression, worry, and BMI (in AN) between the athlete and non-athlete groups at treatment admission. Meng's test of differences (Meng, Rosenthal, \& Rubin, 1992) was also conducted to test for differences among zero-order correlations of ED symptomatology, depression, worry, and psychosocial functioning between groups.

\section{Study 1: Results}

Athlete patients had significantly lower ED symptomatology and depression than non-athlete patients at treatment admission ( $p$ s $<.01$; see Table 2$)$. ED impairment, worry, psychosocial functioning, and BMI (in Anorexia) did not significantly differ between groups at treatment admission ( $p s>.1$ ). Higher ED symptomatology was more strongly associated with higher psychosocial functioning among athlete patients compared to non-athlete patients $(z(167)=3.37, p<.001)$, and higher ED symptomatology was marginally but not significantly associated with higher depression among athletes compared to non-athletes $(z(167)=1.92$, 


\section{Table 2 Study 1: Independent t-Tests Comparing Athlete Patients to Non-Athlete Patients at Admission in Measures of ED Symptomatology and Impairment, Depression, Worry, and BMI in AN Using PSM}

\begin{tabular}{|c|c|c|c|c|c|}
\hline & $\begin{array}{l}\text { Athlete, } \\
M \text { (SD) }\end{array}$ & $\begin{array}{c}\text { Non-Athlete, } \\
M(S D)\end{array}$ & t-value & Sig & $n$ \\
\hline \multicolumn{6}{|c|}{ ED Symptomatology } \\
\hline Admission & $3.37(1.60)$ & $4.00(1.51)$ & 2.59 & $p=.01$ & 167 \\
\hline \multicolumn{6}{|c|}{ ED Impairment } \\
\hline Admission & $29.96(12.54)$ & $33.54(11.06)$ & 1.68 & $p=.10$ & 123 \\
\hline \multicolumn{6}{|l|}{ Depression } \\
\hline Admission & 26.38 (13.97) & $33.42(14.11)$ & -2.68 & $p<.01$ & 127 \\
\hline \multicolumn{6}{|l|}{ Worry } \\
\hline Admission & $57.52(14.14)$ & $61.44(12.38)$ & -1.02 & $p=.31$ & 82 \\
\hline \multicolumn{6}{|c|}{ Psychosocial Functioning } \\
\hline Admission & $2.13(.79)$ & $2.44(1.00)$ & 1.25 & $p=.22$ & 64 \\
\hline \multicolumn{6}{|l|}{ BMI (in AN) } \\
\hline Admission & $17.74(2.64)$ & $17.55(2.49)$ & .38 & $p=.71$ & 108 \\
\hline
\end{tabular}

Note. Differences in $n$ s were due to the implementation of outcomes measures at different time points within the treatment center. PSM=Propensity Score Matching; ED Symptomatology= Eating Disorder Examination Questionnaire; ED Impairment = Clinical Impairment Assessment; Depression $=$ Beck Depression Inventory II; Worry = Penn State Worry Questionnaire; BMI = body mass index.

$p=.054$; see Table 3$)$. ED symptomatology was not more strongly associated with worry in athletes than non-athletes $(r(167)=-1.59, p=.112)$.

\section{Study 2: Methods}

\section{Participants}

Participants were 39 male and female athletes and 26 male and female non-athletes engaged in the same residential or PHP treatment program as those in Study 1. Study 2 was conducted between January and December of 2016 and included new outcome measures not previously utilized by the treatment center. Treatments for both groups did not differ from those in Study 1.

\section{Procedures}

This study was approved by the Institutional Review Board at Washington University in St. Louis. Participants (combined group $N=65$ ) completed the following assessments at the beginning of treatment via paper and pencil: Eating Pathology Symptoms Inventory (EPSI; Forbush et al., 2013); Obsessive Compulsive Inventory Revised (OCI-R; Foa et al., 2002); and Compulsive Exercise Test (CET; Taranis et al., 2011). 
Table 3 Meng's Test of Differences in Zero-Order Correlations in Studies 1 and 2

\begin{tabular}{lcccccc}
\hline & $\begin{array}{c}\text { ED } \\
\text { Symptoms } \\
\text { in Athletes }\end{array}$ & $\begin{array}{c}\text { ED } \\
\text { Symptoms in } \\
\text { Non-Athletes }\end{array}$ & $\begin{array}{c}\text { ED } \\
\text { Symptoms in } \\
\text { Full Sample }\end{array}$ & z-value & sig & $\boldsymbol{n}$ \\
\hline $\begin{array}{l}\text { Psychosocial } \\
\text { Functioning }\end{array}$ & .72 & .57 & .68 & 3.37 & $p<.001$ & 167 \\
Worry & .64 & .71 & .66 & -1.59 & $p=.11$ & 167 \\
Depression & .75 & .68 & .74 & 1.92 & $p=.05$ & 167 \\
OCD Symptoms & .51 & .73 & .55 & -2.56 & $p<.05$ & 65 \\
Compulsive & .60 & .84 & .63 & -3.72 & $p<.001$ & 65 \\
Exercise & & & & & & \\
\hline
\end{tabular}

Note . ED Symptoms = Eating Disorder Examination Questionnaire; Psychosocial Functioning = World Health Disability Assessment Schedule 2.0; Worry = Penn State Worry Questionnaire; Depression = Beck Depression Inventory II; OCD Symptoms = Obsessive-Compulsive Inventory; Compulsive Exercise = Compulsive Exercise Test.

\section{Outcome Measures}

Eating Pathology Symptoms Questionnaire (EPSI; Forbush et al., 2013) was developed to serve as a comprehensive measure of eating pathology and consists of 45 items. The EPSI was added to the battery of assessments utilized by the treatment program to overcome limitations of other ED measures within specific populations, such as men or overweight individuals (Forbush et al., 2013). The eight subscales of the EPSI were used and calculated by summing items within each subscale: body dissatisfaction, binge eating, cognitive restraint, purging, restricting, exercise, negative attitudes toward obesity, and muscle building. The EPSI has been found to have good to excellent convergent and discriminant validity, excellent internal consistency, and acceptable to good test-retest reliability (Forbush et al., 2013). Cronbach's $\alpha$ was .91 for the present study.

Obsessive Compulsive Inventory Revised (OCI-R; Foa et al., 2002) is an 18-item self-report measure, shortened from the original 42-item measure, designed to assess symptoms of OCD. A total score is yielded by summing the six subscales (washing, checking, ordering, obsessing, hoarding, and neutralizing), and higher scores indicate more symptoms of OCD. The OCI-R has good to excellent convergent validity, test-retest reliability, and internal consistency (Foa et al., 2002). Cronbach's $\alpha$ was .89 in the current study.

Compulsive Exercise Test (CET; Taranis et al., 2011) was developed to assess the factors maintaining or operating compulsive exercise in EDs. The CET has 24 items that make up five subscales: avoidance and rule-driven behavior, weight control exercise, mood improvement, lack of exercise enjoyment, and exercise rigidity. Higher global CET scores (calculated by summing the five mean subscale scores) indicate greater compulsive exercise. The CET has demonstrated high internal consistency and concurrent and convergent validity (Taranis et al., 2011). Cronbach's $\alpha$ was .93 in the current study. 


\section{Analyses}

Propensity score matching (PSM; Rosenbaum \& Rubin, 1983) was used to create a final data set $(N=65)$ that accounted for length of stay and age of each treatment group (athletes and non-athletes; see Table 4 for a description of the sample). After using PSM, independent sample t-tests were conducted to test for differences between the athlete and non-athlete groups for the three outcome measures: eating disorder pathology, obsessive-compulsive symptoms, and compulsive exercise at treatment admission. Meng's test of differences was also conducted to test for differences among zero order correlations of ED pathology, obsessive-compulsive symptoms, and compulsive exercise between groups.

\section{Table 4 Study 2: Demographic and Clinical Characteristics of Athlete and Non-Athlete Patients Using PSM}

\begin{tabular}{lc}
\hline & Total (\%) \\
\cline { 2 - 2 } & $\boldsymbol{n}=\mathbf{6 5}$ \\
\hline Gender, $n(\%)$ & $57(87.7)$ \\
Female & $8(12.3)$ \\
Male & $60(93.8)$ \\
European American, $n(\%)$ & \\
ED Diagnoses, $n(\%)$ & $44(67.7)$ \\
AN & $8(12.3)$ \\
BN & $0(0)$ \\
EDNOS & $6(9.2)$ \\
OSFED & $5(7.7)$ \\
BED & $1(1.5)$ \\
ARFID & $1(1.5)$ \\
Rumination & $56.91(25.46)$ \\
LOS in days, $M(S D)$ & 13 to 107 days \\
LOS range & $19.03(4.73)$ \\
Age in years, $M(S D)$ & 10 to 33 years \\
Age range & $3.97(4.73)$ \\
Duration of ED in years, $M(S D)$ & 0 to 21 years \\
Duration of ED range & $26(40)$ \\
Admit BMI $<18.5, n(\%)$ & \\
\hline
\end{tabular}

Note. PSM = Propensity Score Matching; AN = Anorexia Nervosa; $\mathrm{BN}=$ Bulimia Nervosa; EDNOS = Eating Disorder Not Otherwise Specified; OSFED = Other Specified Feeding or Eating Disorder; $\mathrm{BED}=$ Binge Eating Disorder; ARFID $=$ Avoidant Restrictive Food Intake Disorder; Rumination $=$ Rumination Disorder; LOS =Length of Stay in days for the total number of days in either residential and/or partial hospitalization programming; admit $\mathrm{BMI}=$ Admission Body Mass Index. 


\section{Study 2: Results}

No significant differences were found in ED pathology, obsessive-compulsive symptoms, or compulsive exercise between athlete and non-athlete patients at treatment admission ( $p$ s $>.08$; see Table 5). Higher ED symptomatology was more strongly associated with higher obsessive-compulsive symptoms among nonathlete patients compared to athlete patients $(z(167)=-2.56, p<.05$; see Table 3$)$. Interestingly, higher ED pathology was more strongly associated with higher compulsive exercise among non-athletes compared to athletes $(z(167)=-3.72$, $p<.001$; see Table 3).

Table 5 Study 2: Independent t-Tests Comparing Athlete Patients to Non-Athlete Patients at Admission in Eating-Related Pathology, OCD, and Compulsive Exercise Using PSM

\begin{tabular}{|c|c|c|c|c|c|}
\hline & $\begin{array}{l}\text { Athlete, } \\
M \text { (SD) }\end{array}$ & $\begin{array}{c}\text { Non-Athlete, } \\
M(S D)\end{array}$ & t-Value & sig & $n$ \\
\hline \multicolumn{6}{|c|}{ Body Dissatisfaction } \\
\hline Admission & $16.60(7.84)$ & $18.09(9.18)$ & -.65 & $p=.52$ & 57 \\
\hline \multicolumn{6}{|l|}{ Binge Eating } \\
\hline Admission & $11.23(11.35)$ & $11.14(9.90)$ & .03 & $p=.98$ & 57 \\
\hline \multicolumn{6}{|c|}{ Cognitive Restraint } \\
\hline Admission & $8.43(3.39)$ & $8.36(3.84)$ & .07 & $p=.95$ & 57 \\
\hline \multicolumn{6}{|l|}{ Purging } \\
\hline Admission & $3.14(4.09)$ & $4.86(4.87)$ & -.1 .45 & $p=.16$ & 57 \\
\hline \multicolumn{6}{|l|}{ Restricting } \\
\hline Admission & $11.23(6.70)$ & $11.73(7.42)$ & .67 & $p=.79$ & 57 \\
\hline \multicolumn{6}{|l|}{ Exercise } \\
\hline Admission & 11.97 (6.77) & $9.09(7.50)$ & .62 & $p=.14$ & 57 \\
\hline \multicolumn{6}{|c|}{$\begin{array}{l}\text { Negative Attitudes } \\
\text { toward Obesity }\end{array}$} \\
\hline Admission & $8.14(6.32)$ & $7.68(6.97)$ & .63 & $p=.80$ & 57 \\
\hline \multicolumn{6}{|c|}{ Muscle Building } \\
\hline Admission & $4.86(4.75)$ & $2.82(3.06)$ & 1.79 & $p=.08$ & 57 \\
\hline \multicolumn{6}{|c|}{ OCD Symptoms } \\
\hline Admission & 13.17 (10.91) & $18.36(13.81)$ & .60 & $p=.12$ & 57 \\
\hline \multicolumn{6}{|c|}{ Compulsive Exercise } \\
\hline Admission & $13.92(4.36)$ & $13.71(5.13)$ & .16 & $p=.87$ & 57 \\
\hline
\end{tabular}

Note. Body Dissatisfaction, Binge Eating, Cognitive Restraint, Purging, Restricting, Exercise, Negative Attitudes toward Obesity, and Muscle Building comprise the eight Eating Pathology Symptoms Inventory subscales; OCD Symptoms = Obsessive Compulsive Inventory-Revised; Compulsive Exercise $=$ Compulsive Exercise Test . 


\section{Discussion}

The broad aim of this study was to test if athlete patients differed from non-athlete patients in ED symptomatology and related measures upon admission to an intensive ED treatment center. More specifically, the study's objective was to identify any differences that may occur between athletes and non-athletes to better understand the role of EDs in sport. Eating disorder symptomatology was found to be significantly lower in athletes than non-athletes. Eating disorder impairment (i.e., social impairment from EDs as measured by the CIA) and ED pathology (i.e., ED symptoms and thought processes as measured by the EPSI) did not differ between the two groups, suggesting that athletes and non-athletes with EDs have similar levels of impairment and pathology, even without high levels of reported ED symptoms.

Findings in the current study suggest that there may be certain ED symptoms which cause impairment in athletes that are not traditionally assessed in clinical ED settings, such as weight pressures in sport or sport-specific body dissatisfaction (De Bruin, Oudejans, Bakker, \& Woertman, 2011; Galli, Reel, Petrie, Greenleaf, \& Carter, 2011; Reel, SooHoo, Petrie, Greenleaf, \& Carter, 2010). As noted above, the underreporting of ED symptoms by athletes on self-reported questionnaires is not uncommon (Sundgot-Borgen, 1993) and may explain the differences found in the current study. Unfortunately, clinical interviews, which lead to more accurate accounts of ED symptoms in athletes (Sundgot-Borgen, 1993), are often unfeasible in clinical settings given limitations of factors such as time, expense, and staffing. Additional research is called for to investigate alternative ED symptoms in athletes (such as weight pressures in sport or sport-specific body dissatisfaction) and to better understand how ED symptoms and cognitions in athletes are experienced.

Depression was also found to significantly differ between athlete and nonathlete groups. Athlete patients had significantly lower depression than non-athlete patients upon treatment admission. This finding is supported by previous research (Hainline, 2015) and suggests that certain components of sport may be protective against depression. Interestingly, however, higher ED symptomatology was associated with higher depression among athletes more strongly than in non-athletes. This finding indicates that athletes who are depressed are more likely to have higher ED pathology than non-athletes, or that athletes who have high ED pathology are more likely to be depressed than non-athletes. Therefore, depression should be targeted in ED treatment in both athlete and non-athlete patients, and athletes with high levels of ED symptomatology should especially be assessed and treated for depression to effectively aid in the reduction of ED symptoms.

Worry, psychosocial functioning, and OCD symptoms did not differ between athletes and non-athletes. Higher levels of ED symptomatology, however, were associated with higher levels of psychosocial functioning among athletes more strongly than non-athletes. One possible explanation for this finding is that aspects of sport (e.g., social relationships with sport family, support and oversight from sport personnel) may allow for improved psychosocial functioning for athletes compared to non-athletes without such relational opportunities. Higher levels of ED symptomatology were also marginally related to higher levels of obsessivecompulsive symptoms in non-athletes compared to athletes, suggesting that obsessive-compulsive symptoms may have a relationship to EDs in non-athletes, 
but not in athletes when ED symptoms are more severe. This finding is reasonable in the context of viewing sport as either a protective barrier against OCD or as a maintaining factor in OCD, with the latter theory assuming that sports are fulfilling the obsessions or compulsions in athletes without awareness of a problem. However, a paucity of research regarding these variables make it difficult to make confident conclusions. Examining the relationship between OCD and sport, and how sport may influence the development or presentation of OCD, is warranted.

Interestingly, compulsive exercise did not significantly differ between groups. Higher levels of ED symptomatology, however, were associated with higher levels of compulsive exercise in non-athletes more strongly than in athletes. Though future research is needed to understand the mechanisms behind this construct in athletes, it is possible that exercise in the form of intensive training for sport, even if compulsive, is serving a more adaptive function in athletes than non-athletes. As a result, athletes and coaches may see compulsive exercise as a demonstration of a highly dedicated athlete rather than as problematic (De Bruin et al., 2007; Jones et al., 2005).

Finally, BMI in patients with AN did not differ between groups. Though elite athletes tend to have lower BMI's than non-elite athletes and control groups (Nemati, Rahmani-nia, \& Mohebbi, 2018; Neves, Meireles, de Carvalho, Almeida, \& Ferreira, 2016), our findings may be explained by the resources available to some athletes. For example, many athletes have access to weight monitoring and meal planning with a sports dietitian, performance decrements observed by a strength and conditioning coach, and/or changes in medical stability identified by a sports medicine physician. It is possible that these resources allow athletes to be identified with an ED earlier than non-athletes might be, which may prevent symptoms (e.g., BMI) from further deteriorating. More research is necessary to understand if the resources available to athletes relate to early ED detection or early referral to appropriate levels of ED care.

\section{Limitations}

There were several limitations to this study. First, this study had a low sample size of heterogenous patients. Though heterogeneous samples are common among ED treatment centers and reflect clinical populations (Fewell, Levinson, \& Stark, 2017), results should be interpreted judiciously. Furthermore, because of the low sample size, we may have insufficient power needed to detect significant effects. For example, in Study 2, purging and exercise were approaching marginal significance between groups but did not reach significance (see Table 4). Future research should target a larger sample. Another limitation was the use of self-report questionnaires rather than structured clinical interviews, which could lead to inaccurate reporting as previously noted. Additionally, rather than utilizing structured diagnostic interviews for patient diagnoses, psychiatrists engaged in semi-structured clinical interviews to diagnose EDs as part of the admission process, increasing the potential for inconsistent diagnostic assessments (Miller, Dasher, Collins, Griffiths, \& Brown, 2001). Finally, due to discharges and readmissions within the residential and PHP levels of care, this study did not document the percentages of patients in residential versus PHP care, which could have impacted study findings. For example, patients in the residential level of care may 
have reported significantly higher levels of ED and related symptomatology if the ED was more progressed compared to patients in the PHP level of care. Additionally, patients at different levels of care or phases of recovery may have markedly different awareness of their health status and symptom picture and differing abilities to disclose symptom details with accuracy.

\section{Conclusions}

This study is the first to assess the differences and similarities between athlete and non-athlete patients in an intensive ED treatment setting. We found that ED symptomatology and depression differed between athletes and non-athletes. Impairment related to EDs (i.e., cognitive and interpersonal functioning), worry, OCD symptoms, psychosocial functioning, compulsive exercise, and BMI (in AN) did not significantly differ between the two groups. Interesting findings were presented on the relationships between ED symptoms and psychosocial functioning, OCD symptoms, and compulsive exercise among athletes and non-athletes that had not previously been explored. Additional research should investigate the role of sport and athletic status in EDs. Specifically, continued research is needed to better understand how participation in high-level sport influences the presentation, treatment, and outcome of individuals with EDs.

\section{References}

Aardoom, J.J., Dingemans, A.E., Slof Op't Landt, M.C., \& Van Furth, E.F. (2012). Norms and discriminative validity of the Eating Disorder Examination Questionnaire (EDEQ). Eating Behaviors, 13(4), 305-309. PubMed ID: 23121779 doi:10.1016/j.eatbeh. 2012.09.002

Aimé, A., Guitard, T., \& Grousseaud, L. (2017). Anxiety and eating disorders in adult women. In I.J. Lobera (Ed.), Eating disorders-a paradigm of the biopsychosocial model of illness. InTech. doi:10.5772/65249

Anderson, L.M., Reilly, E.E., Gorrell, S., \& Anderson, D.A. (2016). Running to win or to be thin? An evaluation of body dissatisfaction and eating disorder symptoms among adult runners. Body Image, 17, 43-47. PubMed ID: 26952015 doi:10.1016/j.bodyim.2016. 02.003

Arthur-Cameselle, J., Sossin, K., \& Quatromoni, P. (2017). A qualitative analysis of factors related to eating disorder onset in female collegiate athletes and non-athletes. Eating Disorders, 25(3), 199-215. PubMed ID: 27897463 doi:10.1080/10640266.2016. 1258940

Beck, A.T., Steer, R.A., \& Brown, G.K. (1996). Manual for the beck depression inventoryII. San Antonio, TX: Psychological Corporation.

Bohn, K., \& Fairburn, C.G. (2008). The Clinical Impairment Assessment questionnaire (CIA 3.0). In C.G. Fairburn (Ed.), Cognitive behavior therapy and eating disorders. New York, NY: Guilford Press. doi:10.1007/978-981-287-087-2_85-1

Brewer, B.W., Van Raalte, J.L., \& Linder, D.E. (1993). Athletic identity: Hercules' muscles or Achilles heel? International Journal of Sport Psychology, 24(2), 237-254.

Carey, M., Small, H., Yoong, S.L., Boyes, A., Bisquera, A., \& Sanson-Fisher, R. (2014). Prevalence of comorbid depression and obesity in general practice: A cross-sectional survey. British Journal of General Practice, 64(620), e122-e127. PubMed ID: 24567650 doi:10.3399/bjgp14X677482 
Cromer, L., Kaier, E., Davis, J., Stunk, K., \& Stewart, S.E. (2017). OCD in college athletes. American Journal of Psychiatry, 174(6), 595-597. PubMed ID: 28565946 doi:10. 1176/appi.ajp.2017.16101124

Davis, C., Katzman, D.K., Kaptein, S., Kirsh, C, Brewer, H, Kalmbach, K., . . Kaplan, A.S. (1997). The prevalence of high-level exercise in the eating disorders: Etiological implications. Comprehensive Psychiatry, 38, 321-326. PubMed ID: 9406737 doi:10. 1016/S0010-440X(97)90927-5

Davis, C., Katzman, D.K., \& Kirsh, C. (1999). Compulsive physical activity in adolescents with anorexia nervosa: A psychobehavioral spiral of pathology. Journal of Nervous and Mental Disease, 187, 336-342. PubMed ID: 10379720 doi:10.1097/00005053199906000-00002

De Bruin, A.P., Oudejans, R.R., \& Bakker, F.C. (2007). Dieting and body image in aesthetic sports: A comparison of Dutch female gymnasts and non-aesthetic sport participants. Psychology of Sport and Exercise, 8, 507-520. doi:10.1016/j.psychsport.2006. 10.002

De Bruin, A.P., Oudejans, R.R., Bakker, F.C., \& Woertman, L. (2011). Contextual body image and athletes' disordered eating: The contribution of athletic body image to disordered eating in high performance women athletes. European Eating Disorders Review, 19(3), 201-215. PubMed ID: 21584913 doi:10.1002/erv.1112

De Bruin, A.P.K., Woertman, L., Bakker, F.C., \& Oudejans, R.R. (2009). Weight-related sport motives and girls' body image, weight control behaviors, and self-esteem. Sex Roles, 60(9-10), 628-641. doi:10.1007/s11199-008-9562-8

DiBartolo, P.M., \& Shaffer, C. (2002). A comparison of female college athletes and nonathletes: Eating disorder symptomatology and psychological well-being. Journal of Sport and Exercise Psychology, 24(1), 33-41. doi:10.1123/jsep.24.1.33

Fairburn, C.G. (2008). Cognitive behavior therapy and eating disorders. New York, NY: The Guilford Press.

Fewell, L.K., Levinson, C.A., \& Stark, L. (2017). Depression, worry, and psychosocial functioning predict eating disorder treatment outcomes in a residential and partial hospitalization setting. Eating and Weight Disorders-Studies on Anorexia, Bulimia and Obesity, 22(2), 291-301. doi:10.1007/s40519-016-0357-6

Foa, E.B., Huppert, J.D., Leiberg, S., Langner, R., Kichic, R., Hajcak, G., \& Salkovskis, P.M. (2002). The obsessive-compulsive inventory: Development and validation of a short version. Psychological Assessment, 14(4), 485-496. PubMed ID: 12501574 doi: 10.1037/1040-3590.14.4.485

Forbush, K.T., Wildes, J.E., Pollack, L.O., Dunbar, D., Luo, J., Patterson, K., . . Watson, D. (2013). Development and validation of the Eating Pathology Symptoms Inventory (EPSI). Psychological Assessment, 25(3), 859-878. PubMed ID: 23815116 doi: 10.1037/a0032639

Galli, N., Reel, J.J., Petrie, T., Greenleaf, C., \& Carter, J. (2011). Preliminary development of the weight pressures in sport scale for male athletes. Journal of Sport Behavior, 34(1), 47.

Godart, N.T., Flament, M.F., Perdereau, F., \& Jeammet, P. (2002). Comorbidity between eating disorders and anxiety disorders: A review. International Journal of Eating Disorders, 32(3), 253-270. PubMed ID: 12210640 doi:10.1002/eat.10096

Goodwin, H., Haycraft, E., \& Meyer, C. (2016). Disordered eating, compulsive exercise, and sport participation in a UK adolescent sample. European Eating Disorders Review, 24(4), 304-309. PubMed ID: 26892196 doi:10.1002/erv.2441

Green, M.A., Scott, N.A., Cross, S.E., Liao, K.Y., Hallengren, J.J., Davids, C.M., . . . Jepson, A.J. (2009). Eating disorder behaviours and depression: A minimal relationship beyond social comparison, selfesteem, and body dissatisfaction. Journal of Clinical Psychology, 65, 989-999. PubMed ID: 19388056 doi:10.1002/jclp.20586 
Grilo, C., White, M., \& Masheb, R. (2009). DSM-IV psychiatric disorder comorbidity and its correlates in binge eating disorder. International Journal of Eating Disorders, 42, 228-234. PubMed ID: 18951458 doi:10.1002/eat.20599

Gritti, A., Salvati, T., Catone, G., Pisano, S., Salerno, F., Mastroianni, M., \& Bove, D. (2016). Subject at risk for eating disorders: Study of a population of children aged between 8 and 13 years. Journal of Food and Nutritional Disorders, 5(3), 1-4. doi: 10.4172/2324-9323.1000199

Hainline, B. (2015). Mind, body \& sport: Understanding and supporting student-athlete mental wellness [PowerPoint slides on NCAA Sport Science Institute data].

Harris, E.C., \& Barraclough, B. (1998). Excess mortality of mental disorder. British Journal of Psychiatry, 173(1), 11-53. doi:10.1192/bjp.173.1.11

Hughes, L., \& Leavey, G. (2012). Setting the bar: Athletes and vulnerability to mental illness. The British Journal of Psychiatry, 200(2), 95-96. PubMed ID: 22297587 doi:10.1192/bjp.bp.111.095976

Hulley, A.J., \& Hill, A.H. (2001). Eating disorders and health in elite athlete runners. International Journal of Eating Disorders, 30(3), 312-317. PubMed ID: 11746292 doi:10.1002/eat.1090

Jewett, R., Sabiston, C.M., Brunet, J., O’Loughlin, E.K., Scarapicchia, T., \& O’Loughlin, J. (2014). School sport participation during adolescence and mental health in early adulthood. Journal of Adolescent Health, 55(5), 640-644. PubMed ID: 24928804 doi:10.1016/j.jadohealth.2014.04.018

Jones, R.L., Glintmeyer, N., \& McKenzie, A. (2005). Slim bodies, eating disorders and the coach-athlete relationship. International Review for the Sociology of Sport, 40, 377-391. doi:10.1177/1012690205060231

Kaye, W.H., Bulik, C.M., Thornton, L., Barbarich, N., \& Masters, K. (2004). Comorbidity of anxiety disorders with anorexia and bulimia nervosa. American Journal of Psychiatry, 161(12), 2215-2221. PubMed ID: 15569892 doi:10.1176/appi.ajp.161. 12.2215

Kessler, R.C., Berglund, P., Demler, O., Jin, R., Merikangas, K.R., \& Walters, E.E. (2005). Lifetime prevalence and age-of-onset distributions of DSM-IV disorders in the National Comorbidity Survey Replication. Archives of General Psychiatry, 62(6), 593-602. PubMed ID: 15939837 doi:10.1001/archpsyc.62.6.593

Levinson, C.A., Zerwas, S., Calebs, B., Forbush, K., Kordy, H., Watson, H., . . Bulik, C.M. (2017). The core symptoms of bulimia nervosa, anxiety, and depression: A network analysis. Journal of Abnormal Psychology, 126(3), 340-354. PubMed ID: 28277735 doi:10.1037/abn0000254

Luce, K.H., \& Crowther, J.H. (1999). The reliability of the Eating Disorder Examination Self-report Questionnaire Version (EDE-Q). International Journal of Eating Disorders, 25, 349-351. PubMed ID: 10192002 doi:10.1002/(SICI)1098-108X (199904)25:3<349::AID-EAT15>3.0.CO;2-M

Meng, X.L., Rosenthal, R., \& Rubin, D.B. (1992). Comparing correlated correlation coefficients. Psychological Bulletin, 111(1), 172-175. doi:10.1037/0033-2909.111. 1.172

Meyer, T.J., Miller, M.L., Metzger, R.L., \& Borkovec, T.D. (1990). Development and validation of the Penn State Worry Questionnaire. Behaviour Research and Therapy, 28, 487-495. PubMed ID: 2076086 doi:10.1016/0005-7967(90)90135-6

Miller, P.R., Dasher, R., Collins, R., Griffiths, P., \& Brown, F. (2001). Inpatient diagnostic assessments: 1. Accuracy of structured vs. unstructured interviews. Psychiatry Research, 105(3), 255-264. PubMed ID: 11814544 doi:10.1016/S0165-1781(01) 00317-1

Mond, J.M., Hay, P., Rodger, B., Owen, C., \& Beumont, P.J.V. (2004). Validity of the Eating Disorder Examination (EDE-Q) in screening for eating disorders in community 
samples. Behaviour Research and Therapy, 42, 551-567. PubMed ID: 15033501 doi:10.1016/S0005-7967(03)00161-X

Nemati, D., Rahmani-nia, F., \& Mohebbi, H. (2018). Body dissatisfaction and pathogenic weight loss behaviors among Iranian women with different level of physical activity. PsyArXiy Preprints. doi:10.17605/OSF.IO/6X9AS

Neves, C.M., Meireles, J.F.F., de Carvalho, P.H.B., Almeida, S.S., \& Ferreira, M.E.C. (2016). Body dissatisfaction among artistic gymnasticsadolescent athletes and nonathletes. Revista Brasileira de Cineantropometria e Desempenho Humano, 18(1), 82-92. doi:10.5007/1980-0037.2016v18n1p82

Noetel, M., Miskovic-Wheatley, J., Crosby, R.D., Hay, P., Madden, S., \& Touyz, S. (2016). A clinical profile of compulsive exercise in adolescent patients with anorexia nervosa. Journal of Eating Disorders, 4, 1. doi:10.1186/s40337-016-0090-6

Pearlstein, T. (2016). Eating disorders and comorbidity. Archives of Women's Mental Health, 4(3), 67-78. PubMed ID: 29888799 doi:10.1007/s007370200002

Penas-Lledo, E., Bulik, C.M., Lichtenstein, P., Larsson, H., \& Baker, J.H. (2015). Risk for self-reported anorexia or bulimia nervosa based on drive for thinness and negative affect clusters/dimensions during adolescence: A three-year prospective study of the TChAD cohort. International Journal of Eating Disorders, 48(6), 692-699. PubMed ID: 26013185 doi:10.1002/eat.22431

Plateau, C.R., Arcelus, J., Leung, N., \& Meyer, C. (2017). Female athlete experiences of seeking and receiving treatment for an eating disorder. Eating Disorders, 25(3), 273-277. PubMed ID: 28051927 doi:10.1080/10640266.2016.1269551

Reel, J.J., SooHoo, S., Petrie, T.A., Greenleaf, C., \& Carter, J.E. (2010). Slimming down for sport: Developing a weight pressures in sport measure for female athletes. Journal of Clinical Sport Psychology, 4(2), 99-111. doi:10.1123/jcsp.4.2.99

Rosenbaum, P.R., \& Rubin, D.B. (1983). The central role of the propensity score in observational studies for causal effects. Biometrika, 70(1), 41-55. doi:10.1093/biomet/ 70.1 .41

Ruscio, A.M., Stein, D.J., Chiu, W.T., \& Kessler, R.C. (2010). The epidemiology of obsessive-compulsive disorder in the National Comorbidity Survey Replication. Molecular Psychiatry, 15(1), 53-63. PubMed ID: 18725912 doi:10.1038/mp.2008.94

Stöber, J. (1998). Reliability and validity of two widely-used worry questionnaires: Self-report and self-peer convergence. Personality and Individual Differences, 24, 887-890. doi:10.1016/S0191-8869(97)00232-8

Storch, E.A., Roberti, J.W., \& Roth, D.A. (2004). Factor structure, concurrent validity, and internal consistency of the beck depression inventory-Second edition in a sample of college students. Depression and Anxiety, 19, 187-189. PubMed ID: 15129421 doi: $10.1002 /$ da.20002

Sundgot-Borgen, J. (1993). Prevalence of eating disorders in elite female athletes. International Journal of Sport Nutrition, 3(1), 29-40. PubMed ID: 8499936 doi: 10.1123/ijsn.3.1.29

Sundgot-Borgen, J., \& Torstveit, M.K. (2004). Prevalence of eating disorders in elite athletes is higher than in the general population. Clinical Journal of Sport Medicine, 14(1), 25-32. PubMed ID: 14712163 doi:10.1097/00042752-200401000-00005

Taranis, L., Touyz, S., \& Meyer, C. (2011). Disordered eating and exercise: Development and preliminary validation of the Compulsive Exercise Test (CET). European Eating Disorders Review, 19(3), 256-268. PubMed ID: 21584918 doi:10.1002/erv.1108

Thompson, R.A., \& Sherman, R.T. (1999). Athletes, athletic performance, and eating disorders: Healthier alternatives. Journal of Social Issues, 55(2), 317-337. doi:10. 1111/0022-4537.00118

Thompson, R.A., \& Sherman, R.T. (2010). Eating disorders in sport. New York, NY: Taylor \& Francis. 
Turton, R., Goodwin, H., \& Meyer, C. (2017). Athletic identity, compulsive exercise and eating psychopathology in long-distance runners. Eating Behaviors, 26, 129-132. PubMed ID: 28325645 doi:10.1016/j.eatbeh.2017.03.001

Ustün T.B., Kostanjsek N., Chatterji S., \& Rehm J. (Eds.). (2010). Measuring health and disability: Manual for WHO Disability Assessment Schedule (WHODAS 2.0). Geneva, Switzerland: World Health Organization.

Vannucci, A., Kass, A.E., Sinton, M.M., Aspen, V., Weisman, H., Bailey, J.O., . . T Taylor, C.B. (2012). An examination of the clinical impairment assessment among women at high risk for eating disorder onset. Behaviour Research and Therapy, 50, 407-414. PubMed ID: 22516320 doi:10.1016/j.brat.2012.02.009

Wollenberg, G., Shriver, L.H., \& Gates, G.E. (2015). Comparison of disordered eating symptoms and emotion regulation difficulties between female college athletes and nonathletes. Eating Behaviors, 18, 1-6. PubMed ID: 25841217 doi:10.1016/j.eatbeh.2015. 03.008 